\title{
SNHG 6 promotes the progression of Colon and Rectal adenocarcinoma via miR-101-3p and $\mathrm{Wnt} / \beta$-catenin signaling pathway
}

\author{
Qianwen Shao ${ }^{1}$, Jing Xu ${ }^{2}$, Rong Deng ${ }^{3}$, Wei Wei ${ }^{3}$, Bing Zhou ${ }^{3}$, Chao Yue ${ }^{3}$, Miaoling Zhu ${ }^{4}$ and Haitao Zhu ${ }^{5^{*}}$ (D)
}

\begin{abstract}
Background: Small nucleolar RNA host gene 6 (SNHG6) regulates diverse biological processes in cancers. Potential function of SNHG6 in human colon and rectal adenocarcinoma (CRC) was evaluated.

Methods: Quantitative real-time polymerase chain reaction, MTT assays, Colony formation assays, Transwell assay, Western Blotting and Luciferase reporter assays were performed to measure the biological functions and potential molecular mechanisms of SNHG6 in CRC.

Results: SNHG6 was over-expressed in CRC, and high expression of s SNHG6 were associated with short survival times. We then identified miR-101-3p as an inhibitory target of SNHG6. Knockdown of SNHG6 significantly decreased miR-101-3p expression. Moreover, silenced SNHG6 obviously inhibited CRC cell growth, weakened cell invasion capacity and blocked the Wnt/ $\beta$-catenin signaling pathway.
\end{abstract}

Conclusion: SNHG6 could regulate the progression of CRC via modulating the expression levels of miR-101-3p and the activity of Wnt/ $\beta$-catenin signaling.

Keywords: Small nucleolar RNA host gene 6, ceRNA, Sponging, Target therapy, Cell signal pathway

\section{Background}

According to data of the American Cancer Society over the past decade, colon and rectal adenocarcinoma (CRC) remained the top three common cancer types, with 140, 250 estimated new cases and 50, 630 estimated deaths in 2018 [1]. Surgery seems to be the most effective therapeutic approach, but CRC patients still have about half experience recurrence [2]. Nowadays, cancer survival has improved due to treatment improvements, especially after adopting targeted therapies in CRC [3, 4]. Multiple non-coding RNAs have been proved to play important part in tumor development and progression [5-7].

Long non coding RNAs (lncRNAs) are a class of long non-coding RNAs, who exert significant functions as tumor suppressor or oncogene involving in tumorigenesis [8]. Accumulating evidences have consistently indicated

\footnotetext{
*Correspondence: co87r5@163.com

${ }^{5}$ Colorectal cancer center, general surgery department, Jiangsu province cancer hospital, Affiliated Cancer hospital of NanJing Medical University, Jiangsu Cancer Institute, No 42, Baiziting road, NanJing, Nanjing, Jiangsu, People's Republic of China210009

Full list of author information is available at the end of the article
}

that IncRNAs regulated tumorigenesis at both transcriptional and post-transcriptional level through sponging microRNA [9]. For instance, $\mathrm{Yu} F$ et al. reported that lincRNA-p21 suppressed human hepatic stellate cells activation via miR-17-5p-mediated-Wnt/ $\beta$-catenin pathway [10]. Sun W et al. demonstrated that NEAT1_2 could function as a competing endogenous RNA to regulate AAA domain-containing protein 2 expression by sponging miR-106b-5p in papillary thyroid carcinoma [11]. Thus, the aberrant expression of lncRNAs could offer novel pathways of therapeutic targets for CRC.

In the present study, we revealed that SNHG6 was significantly up-regulated in human CRC tissues and cell lines. Over-expression SNHG6 was associated with shorter survival time. Moreover, we identified microRNA miR-101-3p as a negative regulation target for SNHG6. Silencing of SNHG6 suppressed CRC cells proliferation and invasion vitality. In addition, we uncovered that $\beta$-catenin and TCF4 were inhibitory targets of miR101-3p, and that Wnt/ $\beta$-catenin signaling was inhibited by miR-101-3p over-expression. Taken together, we 
demonstrated that the IncRNA SNHG6 promoted CRC progression via regulating the expression of miR-101-3p and the activity of the Wnt/ $\beta$-catenin signaling pathway.

\section{Methods}

\section{Clinical tissue samples}

This research was conducted according to the World Medical Association Declaration of Helsinki and was approved by the ethics committee of Jiangsu province cancer hospital. Written informed consents were signed by all participants. Fifty five pairs of CRC tissues and adjust normal tissues were obtained from CRC patients in Jiangsu province cancer hospital from January, 2014 to March, 2016. Tumor tissues were diagnosed by pathological examination and all participants received no chemotherapy or radiotherapy before the surgery. Tumor tissues were stored at $-80^{\circ} \mathrm{C}$ for later analysis.

\section{Cell culture and transfection}

CRC cell lines HT29, SW620 and normal human intestinal epithelial cells HIECs were purchased from Shanghai Model Cell Bank (Shanghai, China). Cells were cultured in media Roswell Park Memorial Institute-1640 (RPMI-1640; Invitrogen, Carlsbad, CA, USA) or Dulbecco's Modified Eagle's Medium (DMEM; Gibco, Grand Island, NY, USA) supplemented with $10 \%$ fetal bovine serum (Gibco) at $37^{\circ} \mathrm{C}$ in $5 \% \mathrm{CO}_{2}$.

LncRNA SNHG6 siRNA, negative control (NC) siRNA, miR-101-3p and miR-101-3p mimics were all obtained from Gene-Pharma (Shanghai, China), and were transfected with Lipofectamine 2000 reagent (Invitrogen, Karlsruhe, Germany) according to the manufacturer's instructions. Cells were seeded in 6-well plates at a concentration of $2 \times 10^{5}$ cells/well, and were transfected with siRNA or miRNA when cells reached $40-60 \%$ confluence.

\section{Quantitative real-time polymerase chain reaction analysis (qRT-PCR)}

At $48 \mathrm{~h}$ after transfection, total RNA was extracted from CRC tissues or cells using TRIzol reagent (Invitrogen) according to the manufacturer's manual. Then, cDNA was generated by the PrimeScript RT Reagent Kit and qRT-PCR was performed using SYBR Premix Ex Taq (Takara Biotech, Dalian, China) according to the manufacturer's manual. All qRT-PCR assays was performed on an ABI 7900 system (Applied Biosystems, Foster City, CA, USA). GAPDH or U6 was used as an internal control. Expression fold changes were calculated using $2^{-\Delta \Delta \mathrm{Ct}}$ methods.

The primer sequences were as follows:

SNHG6, forward: 5'-CCTACTGACAACATCGAC GTTGAAG-3' and reverse: 5'-GGAGAAAACGCTTA GCCATACAG-3';
GAPDH, forward: 5'-GGGAGCCAAAAGGGTCAT$3{ }^{\prime}$ and reverse: 5'-GAGTCCTTCCACGATACCAA-3'.

MiR-101-3p, forward: 5'-UACAGUACUGUGAU AACUGA A-3' and reverse: 5'-CAGUUAUCACAGUA CUGUAU U-3';

U6, forward: 5'-GCUUCGGCAGCACAUAUACUA AAAU-3' and reverse: 5' -CGCUUCACGAAUUUGCGU GUCAU-3'.

\section{Luciferase assay}

SNHG6-wild-type (SNHG6-Wt) was contracted by cloning the SNHG6 fragment containing the predicted miR101-3p binding site into a pmirGlO Dual-luciferase miRNA Target Expression Vector (Promega, Madison, WI). SNHG6-mutated-type Vector (SNHG6-Mut) was contracted the same way but cloned SNHG6 fragment containing the mutated miR-101-3p binding site. Then vectors and miRNAs were co-transfected into $293 \mathrm{~T}$ cells at the indicated concentrations using Lipofectamine 2000 (Invitrogen) according to the instructions. After 48 h, luciferase activity was measured using a dual-light luminescent reporter gene assay kit (Promega).

\section{MTT assay}

$1 \times 10^{3}$ CRC cells per well were seeded onto 96-well plate and incubated at $37^{\circ} \mathrm{C}$ containing $5 \% \mathrm{CO} 2$. Then, cells were washed twice with phosphate buffer saline (PBS; Thermo Fisher Scienti c, Waltham, MA) and $20 \mu \mathrm{L}$ of methyl thi- azolyl tetrazolium (MTT; Thermo) solution was added to each well. $2 \mathrm{~h}$ later, cellular viability was detected by measuring the absorbance at $450 \mathrm{~nm}$ after $100 \mu \mathrm{L}$ dimethyl sulfoxide (DMSO; Thermo) added to each well.

\section{Colony formation assay}

$0.5 \times 10^{3}$ CRC cells per well transfected with indicated vector were seeded in six-well plates and cultured for two weeks. Colonies were then fixed in $10 \%$ formaldehyde for $10 \mathrm{~min}$ and stained with $0.5 \%$ crystal violet for $10 \mathrm{~min}$. Finally, the number of visible colonies was counted manually.

\section{Transwell assay}

$1 \times 10^{5} \mathrm{CRC}$ cells transfected with indicated vector were seeded in the upper chambers of $8-\mu \mathrm{m}$ pore size insert in the 24-well Transwell chamber (Costar, Boston, MA, USA) with $100 \mu \mathrm{l}$ serum-free medium. The lower chambers were added with $500 \mu \mathrm{l}$ medium containing $10 \%$ fetal bovine serum. After $48 \mathrm{~h}$, the cells on the surface of the upper membrane were removed with a cotton tip, and the cells under the surface of the lower chamber were fixed with $4 \%$ paraformaldehyde for $20 \mathrm{~min}$, stained with $0.1 \%$ crystal violet for 25 min. Invaded cells were 
counted in five randomly selected high-power fields. Experiments were performed in triplicate.

\section{Western blot analysis}

Cells were lysed using RIPA protein extraction reagent (Beyotime, Shanghai, China). Then $25 \mu \mathrm{g}$ protein extracts were separated by $10 \%$ sodium dodecyl sulfate polyacrylamide gel electrophoresis (SDS-PAGE), and then transferred onto nitrocellulose membranes (Millipore, Billerica, MA). Proteins were detected with specific primary antibodies against $\beta$-catenin, Cyclin D1, Axin2 or GAPDH (Santa Cruz, Dallas, Texas, USA) overnight. Horseradish peroxidaselinked secondary antibodies (Beyotime) were used as the second antibodies. Finally, protein blots were visualized with enhanced chemiluminescent substrate (Thermo).

\section{Statistical analysis}

GraphPad prism software was used for statistical analysis, and data was presented as mean $\pm \mathrm{SD}$ from at least three separate experiments. The significance of differences between groups were estimated by Student's t-test, X2 test or Wilcoxon test as appropriate. $P<0.05$ was considered statistically significant.

\section{Results}

LncRNA SNHG6 were up-regulated in CRC

To explore the function of lncRNA SNHG6 in CRC development, we first investigated SNHG6 expression level in TCGA Data Portal from starBASE v2.0 [12, 13]. As Fig. 1a revealed, the expression of SNHG6 was overexpressed in 12 kinds of cancer tissues compared to the adjust normal tissues. Moreover, SNHG6 expression was significantly higher in CRC tissues compared to normal tissues (Fig. 1b; $P<0.001$ ). To support this conclusion, we detected SNHG6 expression from 55 clinical CRC patient samples by qRT- PCR assays, which was ubiquitously increased compared to adjacent non-tumor tissues (Fig. 1c; $P<0.001$ ). Next, SNHG6 expression was

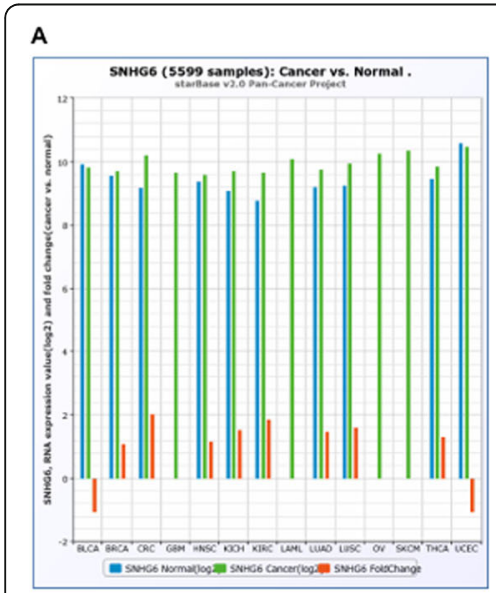

D

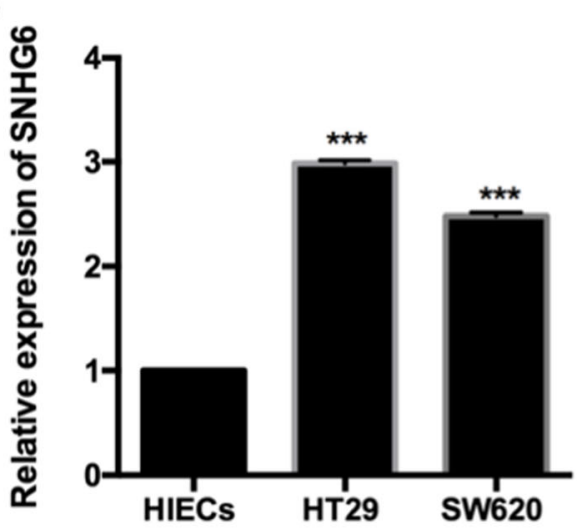

B
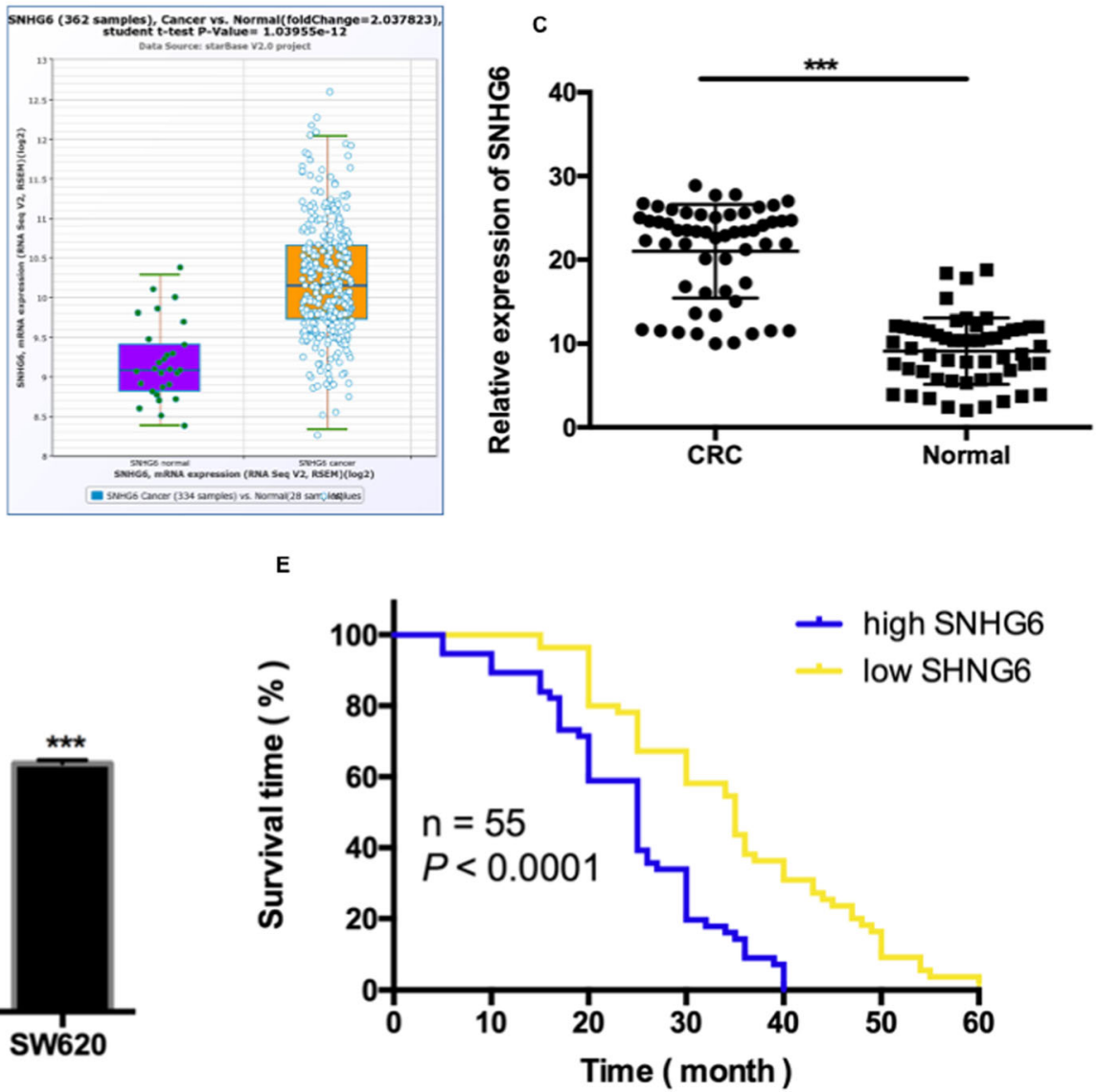

Fig. 1 LnCRNA SNHG6 were up-regulated in CRC. a The expression of SNHG6 among Pan-Cancer including 14 cancer types from The Cancer Genome Atlas (TCGA) Data Portal from starBASE v2.0. b The expression of SNHG6 in CRC or adjacent normal tissues from TCGA Data Portal. c The expression of SNHG6 was determined by qRT-PCR in 55 pairs of CRC tissues comparing with adjacent non-tumour tissues. $\mathbf{d}$ The expression of SNHG6 was examined by qRT-PCR in 2 CRC cell lines (HT29, SW620) and 1 normal human intestinal epithelial cell line HIECs. e High expression of SNHG6 was associated with shorter overall survival of CRC patients. ${ }^{*} P<0.05 ;{ }^{* *} P<0.01$; ${ }^{* *} P<0.001$ 
over-expressed in 2 CRC cells lines (HT29, SW620) comparing to human intestinal epithelial cells HIECs (Fig. 1d; $\mathrm{P}<0.001$ ). In addition, we performed KaplanMeier survival analysis to test the association between SNHG6 expression and the survival in 55 clinical CRC patients. High expression level of SNHG6 caused in much shorter survival time (Fig. 1e; $P<0.0001$ ). Taken together, our results which was consistent with previous data suggested that SNHG6 might play a key role in CRC development and progression.

\section{miR-101-3p was an inhibitory target of IncRNA SNHG6}

Since recent evidences showed that lncRNAs negatively regulated miRNAs expression and activity in cancers $[14,15]$, we doubted if SNHG6 performed the similar molecular mechanisms in CRC. Firstly, we identified miR-101-3p as the potential target of SNHG6 by TCGA
Data Portal from starBASE v2.0. The predicted miRNA target sites of miR-101-3p were showed in Fig. 2a. Ddual-luciferase reporter assay was performed after cotransfected with indicated vector and miRNA into $293 \mathrm{~T}$ cells, respectively. As shown in Fig. 2a, miR-101-3p significantly reduced wild type-SNHG6 luciferase activity, while have no effect on mutant-SNHG6 activity, which indicated that miR-101-3p bine to transcript position of SNHG6. To confirm that target, we knocked down SNHG6 by siRNA transfection. qRT-PCR analysis proved the significant silencing effect in two independent CRC cell lines, HT29 and SW620 cells (Fig. 2b; $P<0.05$ ). Moreover, SNHG6 silencing significantly inhibited the expression of miR-101-3p in both CRC cell lines (Fig. 2c; $P<0.05$ ), but miR-101-3p knock-down didn't cause any change in the expression of SNHG6 (Fig. 2d; $P>0.05$ ). These results supported that SNHG6 expression might be suppressed by

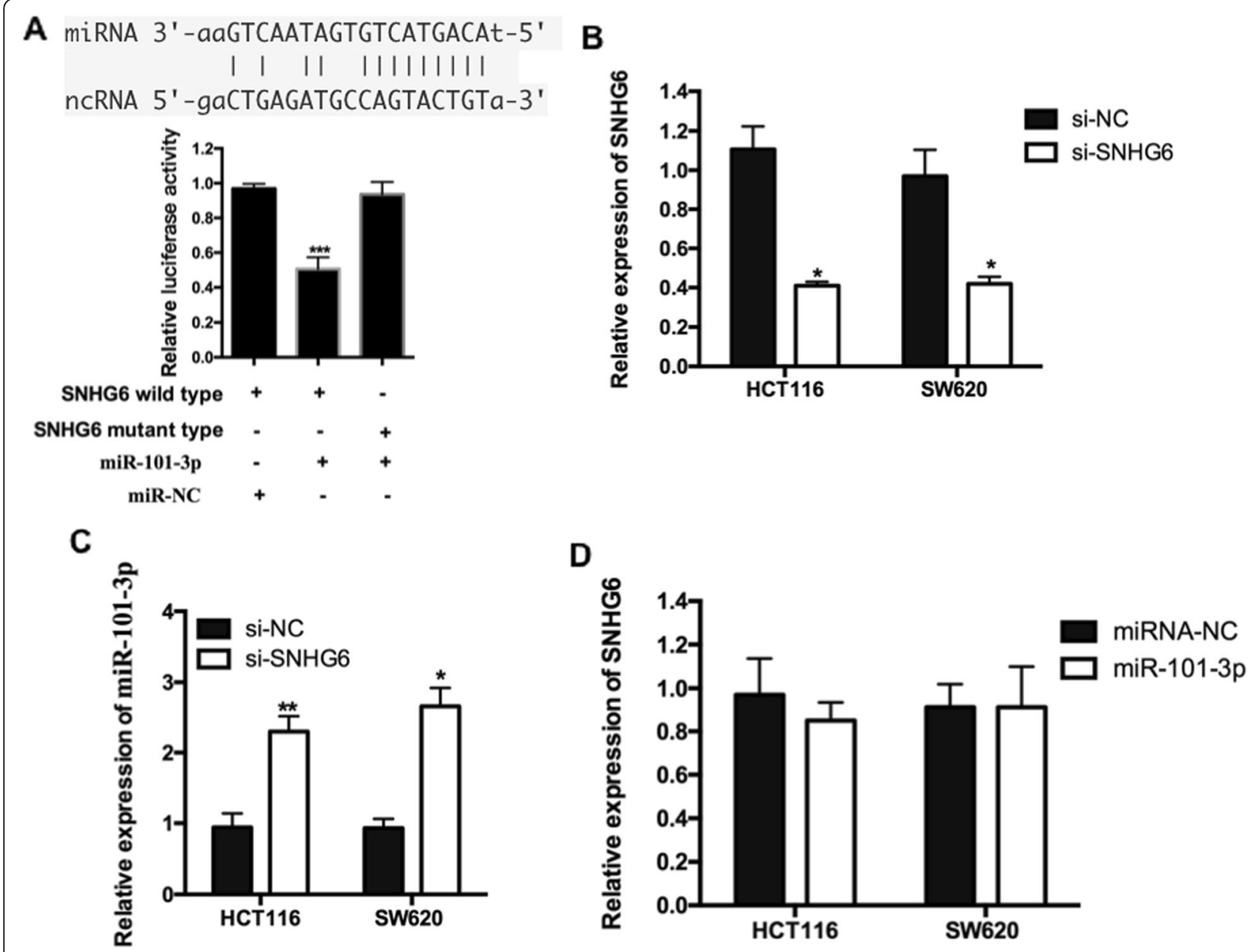

Fig. 2 miR-101-3p was an inhibitory target of IncRNA SNHG6. a Sequence alignment of miR-101-3p with the putative binding sites with in the wild-type regions of SNHG6. Dual-luciferase reporter assay showed that miR-101-3p mimics reduced the intensity of fluorescence in HEK293T cells transfected with SNHG6-Wild type, while had no effect on the SNHG6-mutant vector. b Relative expression of SNHG6 after CRC cells transfected with si-SNHG6 or si-NC. c qRT-PCR analysis of miR-101-3p expression in HCT116 and SW620 cells transfected with si-SNHG6 or si-NC. $\mathbf{d}$ qRT-PCR analysis of SNHG6 expression in HCT116 and SW620 cells transfected with miR-101-3p or miR-NC. ${ }^{* P}<0.05$; ${ }^{* * P}<0.01 ;{ }^{* * * P}<0.001$ 
miR-101-3p, and miR-101-3p was an inhibitory target of SNHG6.

\section{LncRNA SNHG6 promoted CRC cell proliferation and migration}

To determine the function of SNHG6 on CRC cell proliferation, MTT assays were performed on two CRC cell lines that transfected with SNHG6-siRNA, respectively. SNHG6 inhibition significantly inhibited cell proliferation in both CRC cell lines compared to cells transfected with NC-siRNA (Fig. 3a; $P<0.05$ ). We also performed additional assays to confirm the regulation of cell proliferation by SNHG6 and miR-101-3p. Colony formation ability of CRC cells transfected with SNHG6-siRNA were obviously suppressed comparing with cells in NCsiRNA group (Fig. 3b; $\mathrm{P}<0.05$ ). Next, invasion analysis was performed on CRC cells, and we found that CRC cells in SNHG6-siRNA group performed weaker invasion ability compared to NC-siRNA group cells (Fig. 3c; $P<0.05)$. These data collectively indicated that SNHG6 promoted CRC cell proliferation.

miR-101-3p inhibited Wnt/ $\beta$-catenin signaling by targeting $\beta$-catenin

Recent studies showed that miR-101-3p played crucial roles in cancer cell proliferation, and $\beta$-catenin in the

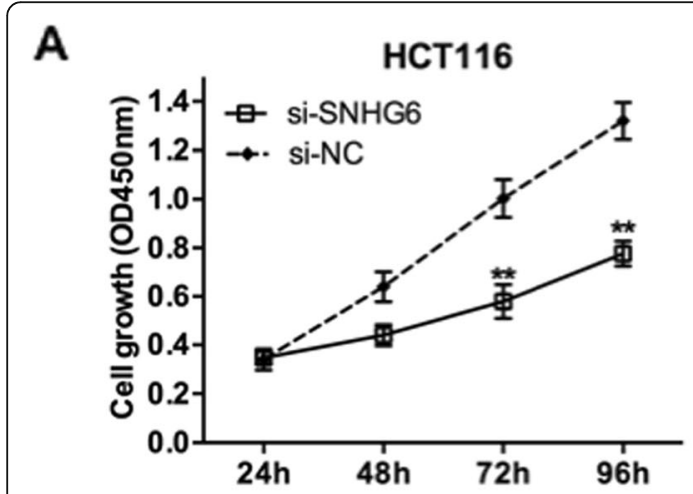

B
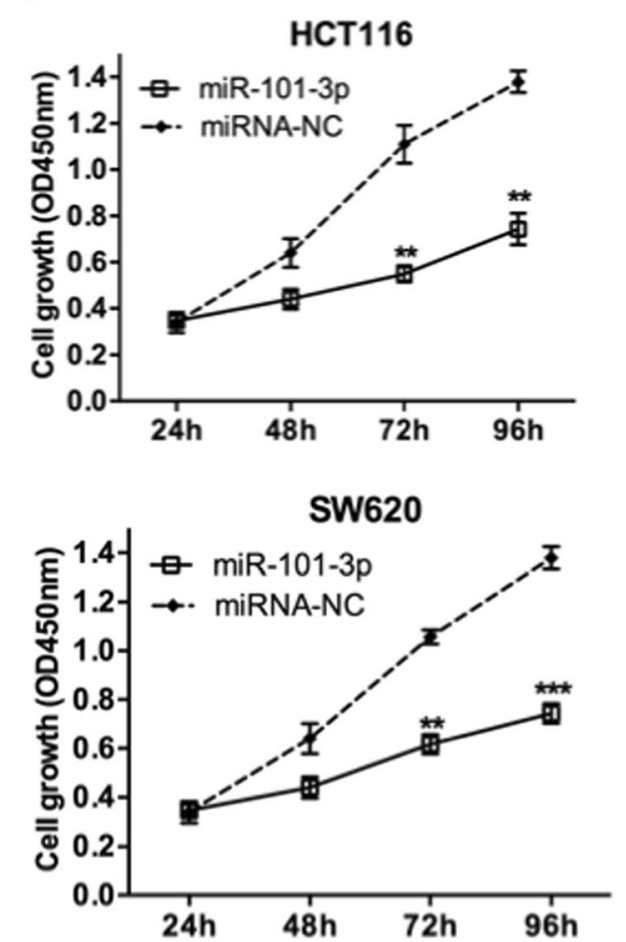

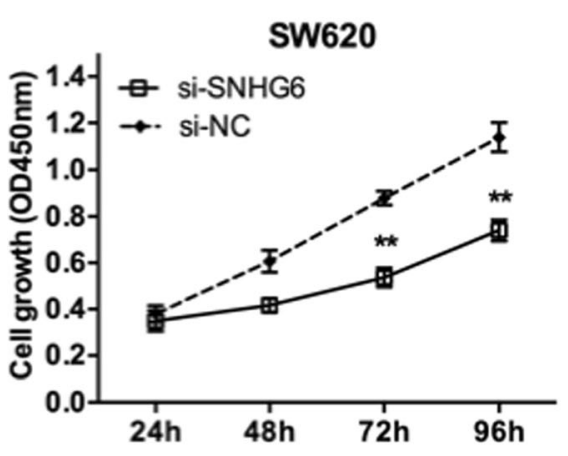

C
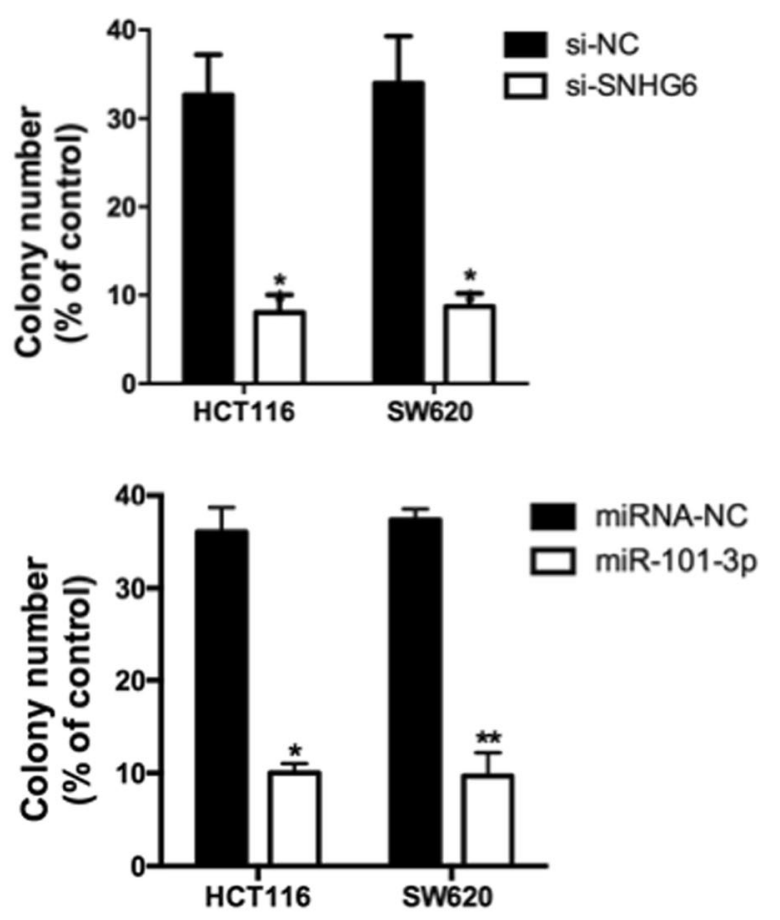

Fig. 3 LncRNA SNHG6 promoted CRC cell proliferation and migration. a Effect of si-SNHG6 on cell proliferation of HCT116 and SW620 cells was detected by MTT assay. $\mathbf{b}$ Effect of si-SNHG6 on colony formation ability of HCT116 and SW620 cells was detected. c Effect of si-SNHG6 on cell invasion ability of HCT116 and SW620 cells was detected. ${ }^{*} \mathrm{P}<0.05$; ${ }^{* *} \mathrm{P}<0.01$ 
Wnt $/ \beta$-catenin signaling pathway were down-regulated after miR-101-3p transfection [16]. To figure out the molecular mechanisms of miR-101-3p oncogenic functions, the luciferase activity of intracellular signal transducer $\beta$-catenin was determined by co-transfecting $\beta$ catenin-Wt or $\beta$-catenin-Mut with miR-101-3p. We noticed that the luciferase activity of $\beta$-catenin was reduced in $\beta$-catenin-Wt group cells (Fig. 4a; $P<0.01$ ). Furthermore, we discovered that the levels of downstream target genes of the $\mathrm{Wnt} / \beta$-catenin signaling pathway, including $\beta$-catenin and c-Myc, were significantly suppressed by miR-101-3p over-expression or SNHG6 knockdown in CRC cells (Fig. 4b). Collectively, our data strongly support the hypothesis that SNHG6 promotes CRC progression and development via miR-101-3p mediated regulation of Wnt/ $\beta$ - catenin signaling (Fig. 4c).

\section{Discussion}

Small nucleolar RNA host gene 6 (SNHG6), a newfound lncRNA located at chromosome 8q13.1, has been demonstrated to be a potential oncogene involved in the initial and development of various cancers, such as breast cancer [17], gastric cancer [18], hepatocellular carcinoma [19], and colorectal adenocarcinoma [20]. Yet, the biological roles and underlying mechanism of SNHG6 in CRC are largely unknown.

Nowadays, lncRNA including SNHG6 has been found to be a sponge for microRNA in multiply tumors. For example, in 2016, Chang L et al. suggested that SNHG6 promoted hepatocellular carcinoma growth and metastasis by endogenous competing miR-101-3p [21]. Then in 2017, Yan K et al. reported that SNHG6 could act as an oncogene in gastric cancer through competitively sponging miR-101-3p and silencing p27 [18]. The mature miRNA microRNA-101-3p (miR-101-3p) has been reported to been associated with carcinogenesis and cancer therapy in several malignancies [22, 23]. In this research, we investigated whether SNHG6 regulate the development and progression of CRC in a similar way. In our investigation, we found that SNHG6 expression was significantly increased in human CRC tissues and cell lines comparing to adjacent normal tissues or normal cells, and it was also corroborated by the analysis of available data in the TCGA database, which investing SNHG6 might play a great role in the progression of CRC. Moreover, we identified miR-101-3p as an inhibitory target of SNHG6 by luciferase reporter assay. Silencing of SNHG6 increased miR-101-3p expression,

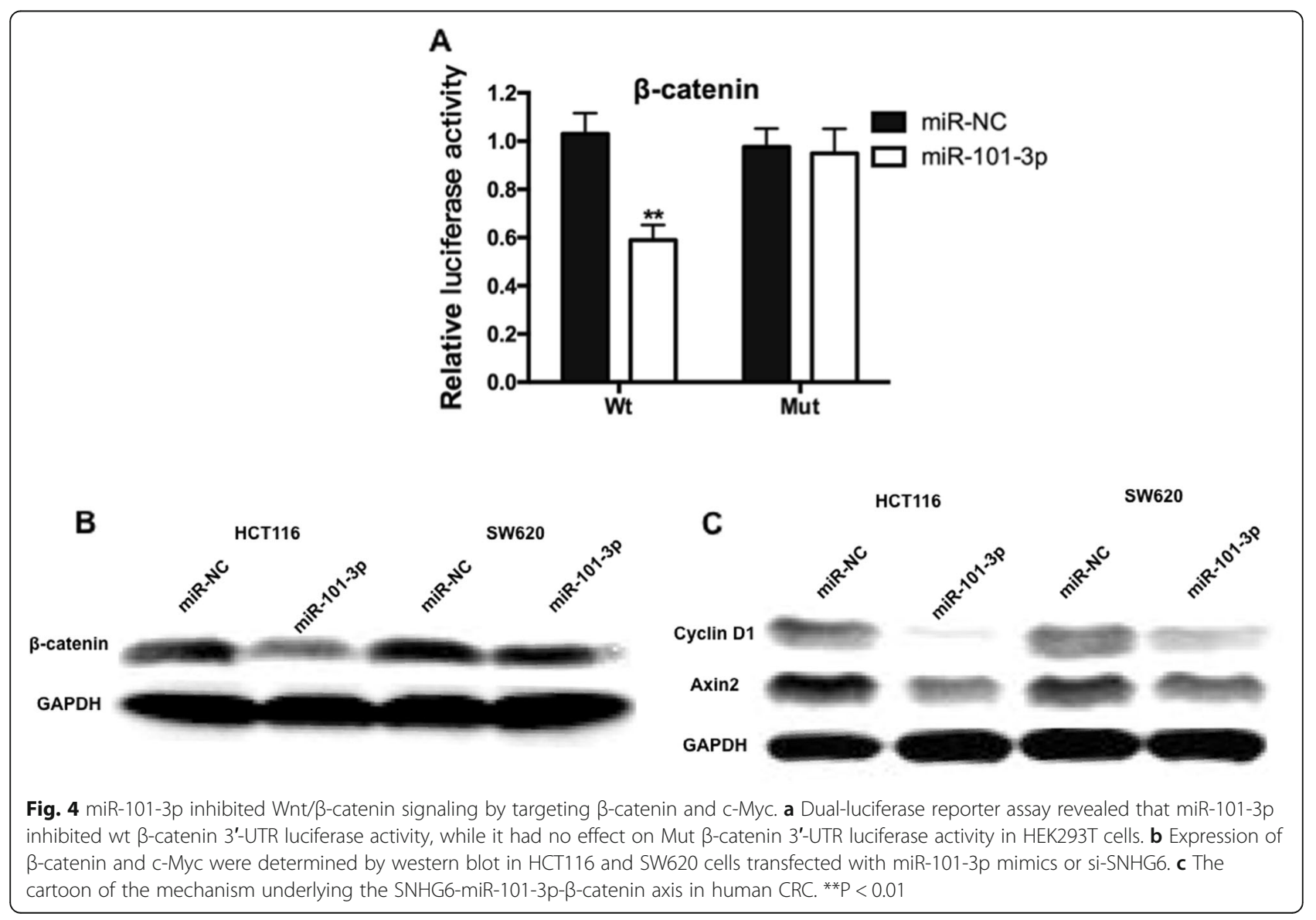


suggesting that miR-101-3p was the downstream of SNHG6. Furthermore, SNHG6 knockdown markedly inhibited CRC cells proliferation and invasion in vitro. Collectively, it documented that SNHG6 promoted carcinogenesis by acting as a miR-101-3p sponge in CRC.

Wnt/ $\beta$-catenin signaling is known to regulate a broad range of cellular processes via regulating the expression of the multifunctional $\beta$-catenin protein, which is a crucial growth stimulatory factor in the $\mathrm{Wnt} / \beta$-catenin pathway, leading to cell proliferation, invasion, differentiation and other signaling pathways [24, 25]. Mutated $\mathrm{Wnt} / \beta$-catenin pathway components are causative to multiple growth-related pathologies and to cancer. Recent studies showed a significant correlation between intracellular signal transducer $\beta$-catenin in $\mathrm{Wnt} / \beta$-catenin signaling pathway and miR-101-3p in tumor progression. For example, SNHG1 have been found to act as an oncogenic lncRNA promoting NSCLC tumorigenesis and progression via miR-101-3p/SOX9/ Wnt/ß-catenin axis [26]. We then sought to confirm this prediction in the context of CRC cells. With dual-luciferase activity assay and western blot assay, the activity of $\mathrm{Wnt} / \beta$-catenin signaling and the protein level of $\beta$-catenin and c-Myc (the downstream target genes of the $W n t / \beta$-catenin signaling pathway) were substantially decreased by miR-101-3p over-expression or SNHG6 knockdown in CRC cells.

\section{Conclusion}

We identified that SNHG6 could act as an oncogenic lncRNA that promoted CRC tumorigenesis and progression via miR-101-3p/Wnt/ $\beta$-catenin axis, which providing a novel potential therapeutic target for the treatment of CRC.

\section{Abbreviations}

CRC: Colon and rectal adenocarcinoma; qRT-PCR: Quantitative real-time polymerase chain reaction analysis; SNHG6: Small nucleolar RNA host gene 6

\section{Acknowledgements}

Not applicable.

\section{Authors' contributions}

SQ and $\mathrm{XJ}$ analyzed and interpreted the patient data regarding the Colon and Rectal Adenocarcinoma. DR,WW and ZM performed cell experiments. ZB and $Y C$ performed experiments in molecular biology. SQ and ZH were the major contributors in writing the manuscript. All authors read and approved the final manuscript.

\section{Funding}

This work was supported by the [Key Project of and Technology Department of Jiangsu Province of China \#1] under Grant [number BL2014092]; [Natural Science Foundation of Jiangsu Province \#2] under Grant [number BK20131438]; [Six talent peaks project in Jiangsu Province \#3] under Grant [number WSW-038]; and [Key Project of Cancer hospital of NanJing Medical University \#4] under Grant. The funding body supported the data collection used in this study. The funding body has no role in the design of the study and analysis and interpretation of data and in writing the manuscript.

Availability of data and materials Not applicable.

\section{Ethics approval and consent to participate}

This research was conducted according to the World Medical Association Declaration of Helsinki and was approved by the ethics committee of Jiangsu province cancer hospital. Written informed consents were signed by all participants.

\section{Consent for publication}

Not applicable.

\section{Competing interests}

This work was supported by the [Key Project of and Technology Department of Jiangsu Province of China \#1] under Grant [number BL2014092]; [Natural Science Foundation of Jiangsu Province \#2] under Grant [number BK20131438]; [Six talent peaks project in Jiangsu Province \#3] under Grant [number WSW-038]; and [Key Project of Cancer hospital of NanJing Medical University \#4] under Grant. The funding body supported the data collection used in this study. The funding body has no role in the design of the study and analysis and interpretation of data and in writing the manuscript. Furthermore, the authors declare that they have no competing interests.

\section{Author details}

'Department of oncology, The First Affiliated Hospital of Nanjing Medical University, Nanjing, Jiangsu, People's Republic of China210029. ${ }^{2}$ Genetic testing center, department of oncology, The First Affiliated Hospital of Nanjing Medical University, Nanjing, Jiangsu, People's Republic of China210029. ${ }^{3}$ General Department, Cancer hospital of NanJing Medical University, Nanjing, Jiangsu, People's Republic of China210009. ${ }^{4}$ Pathology department, Cancer hospital of NanJing Medical University, Nanjing, Jangsu, People's Republic of China210009. ${ }^{5}$ Colorectal cancer center, general surgery department, Jiangsu province cancer hospital, Affiliated Cancer hospital of NanJing Medical University, Jiangsu Cancer Institute, No 42, Baiziting road, NanJing, Nanjing, Jiangsu, People's Republic of China210009.

Received: 30 July 2019 Accepted: 4 September 2019

Published online: 18 September 2019

\section{References}

1. Siegel RL, Miller KD, Jemal A. Cancer statistics, 2018. CA Cancer J Clin. 2018.

2. Siegel RL, Miller KD, Fedewa SA, Ahnen DJ, Meester RG, Barzi A, Jemal A. Colorectal cancer statistics, 2017. CA Cancer J Clin. 2017;67(3):177.

3. Ortega J, Vigil CE, Chodkiewicz C. Current progress in targeted therapy for colorectal cancer. Cancer Control Journal of the Moffitt Cancer Center. 2010; 17(1):7-15.

4. Qureshi A, Verma A, Ross P and Landau D: Colorectal cancer treatment. Bmj Clin Evid 2010(2010)

5. Gutschner T, Hämmerle M, Eissmann M, Hsu J, Kim Y, Hung G, Revenko A, Arun $\mathrm{G}$, Stentrup M, Gross M. The noncoding rna malat1 is a critical regulator of the metastasis phenotype of lung cancer cells. Cancer Res. 2013;73(3):1180.

6. Ostankovitch M, Pyle AM. Noncoding rnas: a story of networks and longdistance relationships. J Mol Biol. 2013:425(19):3577-81.

7. Wang $P, X u e ~ Y$, Han Y, Lin L, Wu C, Xu S, Jiang Z, Xu J, Liu Q, Cao X. The stat3-binding long noncoding ma Inc-dc controls human dendritic cell differentiation. Science. 2014:344(6181):310-3.

8. Yarmishyn AA, Kurochkin IV. Long noncoding rnas: A potential novel class of cancer biomarkers. Frontiers in Genetics. 2015;6(145).

9. Yang L, Froberg JE, Lee JT. Long noncoding rnas: fresh perspectives into the rna world. Trends Biochem Sci. 2014:39(1):35-43.

10. Yu F, Guo Y, Chen B, Shi L, Dong P, Zhou M and Zheng J: Lincrna-p21 inhibits the wnt/ $\beta$-catenin pathway in activated hepatic stellate cells via sponging microrna-17-5p. Cellular Physiologyls\&lsbiochemistry:international Journal of Experimental Cellular Physiology, Biochemistry, \s\&lspharmacology 41(5): 1970, 2017.

11. Sun W, Lan X, Zhang H, Wang Z, Dong W, He L, Zhang T, Zhang P, Liu J, Qin Y. Neat1_2 functions as a competing endogenous rna to regulate atad2 expression by sponging microrna-106b-5p in papillary thyroid cancer. Cell Death Dis. 2018:9(3):380.

12. Yang JH, Li JH, Shao P, Zhou H, Chen YQ, Qu LH. Starbase: a database for exploring microrna-mrna interaction maps from argonaute clip-seq and degradome-seq data. Nucleic Acids Res. 2011;39(Database issue):D202. 
13. Li JH, Liu S, Zhou H, Qu LH, Yang JH. Starbase v2.0: Decoding mirna-cerna, mirna-ncrna and protein-rna interaction networks from large-scale clip-seq data. Nucleic Acids Research. 2014;42(Database issue):D92.

14. Jalali S, Bhartiya D, Lalwani MK, Sivasubbu S, Scaria V. Systematic transcriptome wide analysis of Incrna-mirna interactions. PLoS One. 2013; 8(2):e53823.

15. Paraskevopoulou MD, Georgakilas G, Kostoulas N, Reczko M, Maragkakis M, Dalamagas TM, Hatzigeorgiou AG. Diana-Incbase: experimentally verified and computationally predicted microrna targets on long non-coding rnas. Nucleic Acids Res. 2013;41(Database issue):D239.

16. Wang $H$, Meng $Y$, Cui Q, Qin F, Yang H, Chen Y, Cheng Y, Shi I and Guo Y: Mir-101 targets the ezh2/wnt/ $\beta$-catenin the pathway to promote the osteogenic differentiation of human bone marrow-derived mesenchymal stem cells. Sci Rep 6(36988, 2016.

17. Ding X, Zhang Y, Yang H, Mao W, Chen B, Yang S, Ding X, Zou D, Mo W, He $X$. Long non-coding rnas may serve as biomarkers in breast cancer combined with primary lung cancer. Oncotarget. 2017:8(35):58210.

18. Yan K, Tian J, Shi W, Xia H, Zhu Y. Lncrna snhg6 is associated with poor prognosis of gastric cancer and promotes cell proliferation and emt through epigenetically silencing p27 and sponging mir-101-3p. Cellular Physiology \& Biochemistry International Journal of Experimental Cellular Physiology Biochemistry \& Pharmacology. 2017;42(3):999.

19. Birgani MT, Hajjari M, Shahrisa A, Khoshnevisan A, Shoja Z, Motahari P and Farhangi B: Long non-coding rna snhg6 as a potential biomarker for hepatocellular carcinoma. Pathology and Oncology Research 1): 1-9, 2017.

20. Li M, Bian Z, Yao S, Zhang J, Jin G, Wang X, Yin Y, Huang Z. Up-regulated expression of snhg6 predicts poor prognosis in colorectal cancer. Pathology Research and Practice. 2018.

21. Chang L, Yuan Y, Li C, Guo T, Qi H, Xiao Y, Dong X, Liu Z, Liu Q. Upregulation of snhg6 regulates zeb1 expression by competitively binding mir-101-3p and interacting with upf1 in hepatocellular carcinoma. Cancer Lett. 2016;383(2):183-94.

22. Friedman JM, Liang $\mathrm{G}$, Jones $\mathrm{PA}$. The tumor suppressor microrna-101 becomes an epigenetic player by targeting the polycomb group protein ezh2 in cancer. Cell Cycle. 2009;8(15):2313-4.

23. Friedman JM, Liang G, Liu CC, Wolff EM, Tsai YC, Ye W, Zhou X, Jones PA. The putative tumor suppressor microrna-101 modulates the cancer epigenome by repressing the polycomb group protein ezh2. Cancer Res. 2009:69(6):2623-9.

24. Clevers H, Nusse R. Wnt/ß-catenin signaling and disease. Cell. 2012;149(6): 1192.

25. Niehrs C. The complex world of wnt receptor signalling. Nat Rev Mol Cell Biol. 2012;13(12):767-79.

26. Cui Y, Zhang F, Zhu C, Geng L, Tian T, Liu H. Upregulated Incrna snhg1 contributes to progression of non-small cell lung cancer through inhibition of mir-101-3p and activation of wnt/ $/ 1^{2}$-catenin signaling pathway. Oncotarget. 2017;8(11):17785-94.

\section{Publisher's Note}

Springer Nature remains neutral with regard to jurisdictional claims in published maps and institutional affiliations.

Ready to submit your research? Choose BMC and benefit from:

- fast, convenient online submission

- thorough peer review by experienced researchers in your field

- rapid publication on acceptance

- support for research data, including large and complex data types

- gold Open Access which fosters wider collaboration and increased citations

- maximum visibility for your research: over $100 \mathrm{M}$ website views per year

At $\mathrm{BMC}$, research is always in progress.

Learn more biomedcentral.com/submissions 